\title{
Resonant-Repetitive Combined Control for Stand-Alone Power Supply Units
}

\author{
Alessandro Lidozzi, Luca Solero, \\ Fabio Crescimbini \\ Department of Engineering \\ Roma Tre University, Italy \\ email: alessandro.lidozzi@uniroma3.it
}

\author{
Chao Ji, Pericle Zanchetta \\ Dept. of Electrical and Electronic Engineering \\ University of Nottingham, UK \\ email: pericle.zanchetta@nottingham.ac.uk
}

\begin{abstract}
This paper investigates a combined ResonantRepetitive (RR) control structure for a three phase four leg DC/AC converter power supply. The RR control configuration is composed by a resonant controller tuned at the system fundamental frequency working in conjunction with a plug-in type repetitive controller. The resonant part of the control scheme is used to assure prompt tracking of the inverter output voltage as well to achieve as fast as possible system response to load variations; to this purpose, it is tuned at the fundamental frequency. At the same time, the resonant controller is able to stabilize the system without the necessity of any further additional controller; the repetitive part of the scheme is implemented for the fine regulation at the system harmonic frequencies. The proposed control configuration is used to regulate the power supply output voltage providing very good tracking of the output voltage reference even in the presence of a non-linear load. Experimental validation from a $40 \mathrm{kVA}$ converter prototype is presented to validate the operation of the proposed converter and control.
\end{abstract}

Index Terms - Distributed power generation, DC-AC power converters, harmonic distortion, repetitive control, resonant control, unbalanced loads.

\section{INTRODUCTION}

Distributed generation is being developed worldwide to meet the increasing energy demand as well to reduce the environmental impact of fossil-fuel based centralized generation. Conventional electric power plants have rated power of thousands MVA and are usually located far from the load areas where the energy supply is needed. On the contrary, distributed generation systems are accomplished by using renewable energy generating units (e.g., photovoltaic arrays and wind turbines) which have relatively low power rating and are suitably connected to the power grid at the voltage level of the electricity distribution network, so that the significant investment in electric transmission infrastructure can be substantially reduced. However, due to the intermittent nature of the renewable energy sources and the desire for generating operation with maximum power point tracking, energy storage is usually needed and thereby the various electric power sources are operated in parallel against a common DC link by means of power electronic converters, as schematically shown in Fig. 1.

Hence, a DC-AC converter with 4-wire output is used for connecting the generating system to the distribution network and the user loads. Such a converter is required to provide both active and reactive power output depending on whether it is operated on-grid or off-grid. The off-grid or even standalone mode of operation is the most severe in terms of control stability as well as waveforms total harmonic distortion (THD); in fact, the absence of the grid connection, which is known as low impedance connection, makes the whole generating system easily affected by non-linear 3phase and 1-phase loads. As a consequence, the paper refers to the off-grid mode of operation of the DC-AC converter with 4-wire output in order to investigate, in particular, the control issues.

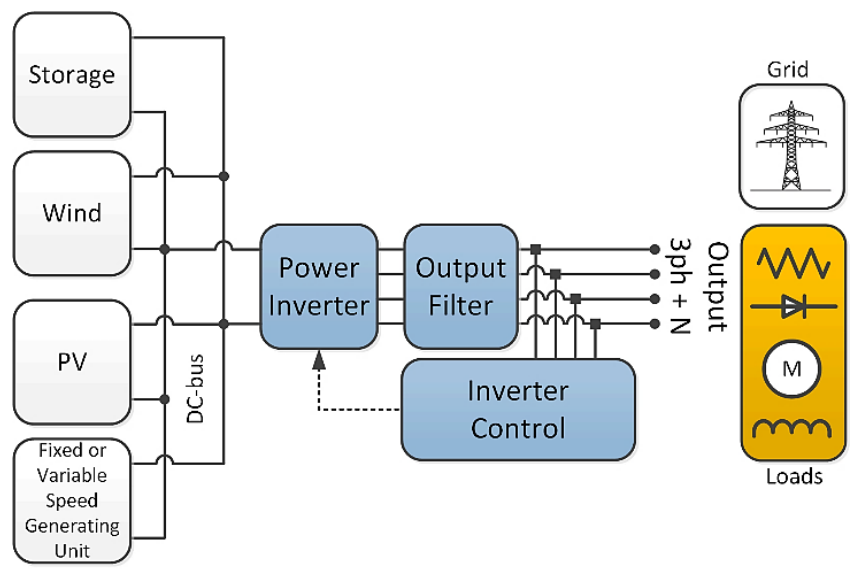

Fig. 1. Example of Distributed Generating System.

The 3-phase 4-leg inverter topologies make use of an additional leg with respect to the conventional 3-phase topologies as it is shown in Fig. 2. The additional leg requires 2 more switches and power diodes as well extra driving circuits and higher complexity in modulation techniques and control strategies. In spite of this, the 4-leg inverter, when properly modulated, can assure the efficient regulation of the output phase voltage also in case of unbalanced and distorted loads. Injection of $3^{\text {rd }}$ harmonic as well SVM techniques are possible without affecting the harmonic content in phase-to-neutral voltage [1]; further, the neutral current flows through the added leg and no oversizing is required for the DC-link capacitors.

Emerging control techniques, in the field of power electronics for both on-grid and off-grid electric generating systems, are the resonant control (RSC) and the repetitive control (RC) which have found significant space in the last 
decade literature. Both $\mathrm{RC}$ and RSC are based on the Internal Model Principle. RSC is directly able to compensate any sinusoidal disturbance even if not multiple of the fundamental harmonic. More details are shown in Section II. RC uses the error signal of previous cycle to improve the performance of current cycle and it is described in details in Section III.

In practical applications, the control system can become unstable where high order RSCs are used to compensate the harmonic distortion introduced by the loads. It causes the reduction of the overall control accuracy due to the limited number of harmonic controllers that can be used. This behavior is strongly related to the output power filter phase lagging which heavily depends on the load condition. At the same time, the repetitive control is not able to ensure system stability by itself and it needs an additional control action. Also with RC the phase behavior of the whole system is of critical importance.

Some solutions for resonant controller phase control have been shown in literature [2], where the resonant controller ideal transfer function is extended including phase compensation. However, the proposed control structure does not consider the influence of the load variation in the tuning process and during the inverter operation. Phase control in $\mathrm{RC}$ has been preliminary investigated in [3].

The paper first discusses both the RSC and the RC basic characteristics and then the combination of the two control structures (RR) is investigated with reference to a 4-leg DC$\mathrm{AC}$ converter for power supply of unbalanced and nonlinear loads.

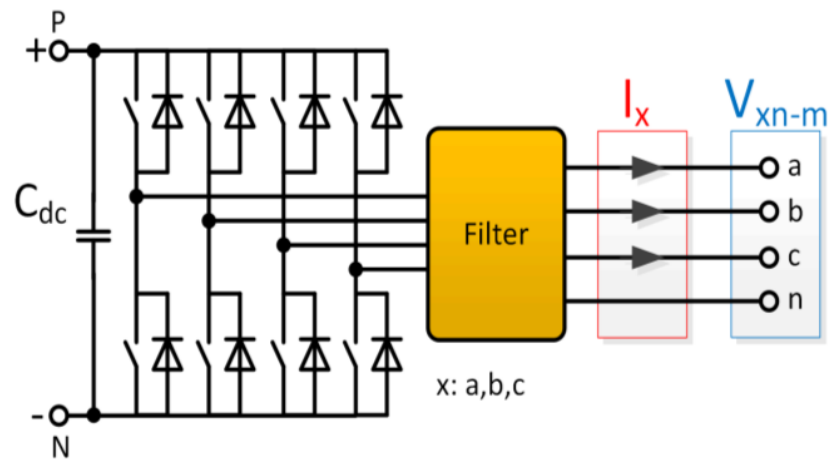

Fig. 2. 3-Phase 4-Leg Inverter. x: a, b, c line. n: neutral. m: measured.

\section{RESONANT CONTROL}

Resonant controllers can be synthesized through different analytical expressions, the ideal form, the approximated form and the full real form. Each RSC can be combined with others to form the so-called Multi Resonant Control Structure [4], where several harmonics can be compensated.

The RSC ideal form is very simple and it is shown in (1)

$$
G_{R S C i}(s)=\frac{k_{i r} s}{s^{2}+\omega_{0}^{2}}
$$

where $\mathrm{k}_{\mathrm{ir}}$ is the controller gain and $\omega_{0}$ the resonance frequency. However, the ideal form should be avoided due to its infinite gain at the resonance frequency, which makes the transfer function difficult to be digitally implemented on industrial grade Digital Signal Processors (DSPs) working with fixed or single precision floating-point arithmetic. Moreover, it does not allow selecting the appropriate controller gain and width, resulting in a strong reduction of control design flexibility.

An alternative RSC form can be achieved by the approximated full-structure expressed as in (2)

$$
G_{R S C a}(s)=\frac{2 k_{i r} \omega_{c r} s}{s^{2}+2 \omega_{c r} s+\omega_{0}^{2}}
$$

where $\omega_{\mathrm{cr}}$ is the controller width. However, the validity of the approximation, $\omega_{\mathrm{cr}}<<\omega_{0}$, must be verified every time the controller gains are changed.

In order to provide a more general RSC form, the full real resonant controller having the transfer function reported in (3) can be considered.

$$
G_{R S C f}(s)=\frac{2 k_{i r}\left(\omega_{c r} s+\omega_{c r}^{2}\right)}{s^{2}+2 \omega_{c r} s+\omega_{c r}^{2}+\omega_{0}^{2}}
$$

As depicted in [5] RSC allows also regulating the controller phase at the resonant frequency, increasing the design flexibility with three independent degrees of freedom: controller gain, width and phase (4).

$$
G_{R S C f \vartheta}(s)=\frac{2 k_{i r} \omega_{c r}\left(s \cos (\vartheta)+\omega_{c r}-\omega_{0} \sin (\vartheta)\right)}{s^{2}+2 \omega_{c r} s+\left(\omega_{c r}^{2}+\omega_{0}^{2}\right)}
$$

Each RSC can be part of a more complex structure when more than one RSC are combined to provide compensation at different frequencies. Moreover, the RSC requires the addition of an integral term to the whole transfer function in order to effectively regulate the DC component. As in more conventional control structures, a proportional gain can be inserted in the transfer function to increase the dynamic behavior. The general control scheme related to the aforementioned control topologies is shown in Fig. 3a where a multi resonant structure can include both proportional gain $\mathrm{K}_{\mathrm{p}}$ and integral action $\mathrm{K}_{\mathrm{i}} / \mathrm{s}$; whereas, Fig. $3 \mathrm{~b}$ depicts the bode plots for the previously described RSC based control alternatives. Resonant controller design can be carried out using well-known techniques as in [6]. However, the maximum harmonic order in the multi-resonant control structure is limited by the load-filter interaction, which causes the decreasing of resonant controllers' magnitude and phase. In particular, the phase variation causes the reduction of the system phase margin and proper compensation strategies must be implemented in the control algorithm [5]. 


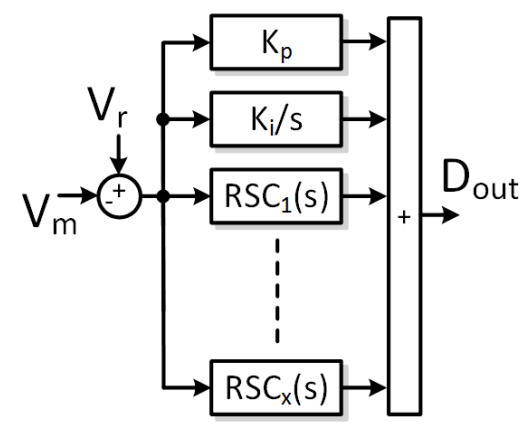

(a)

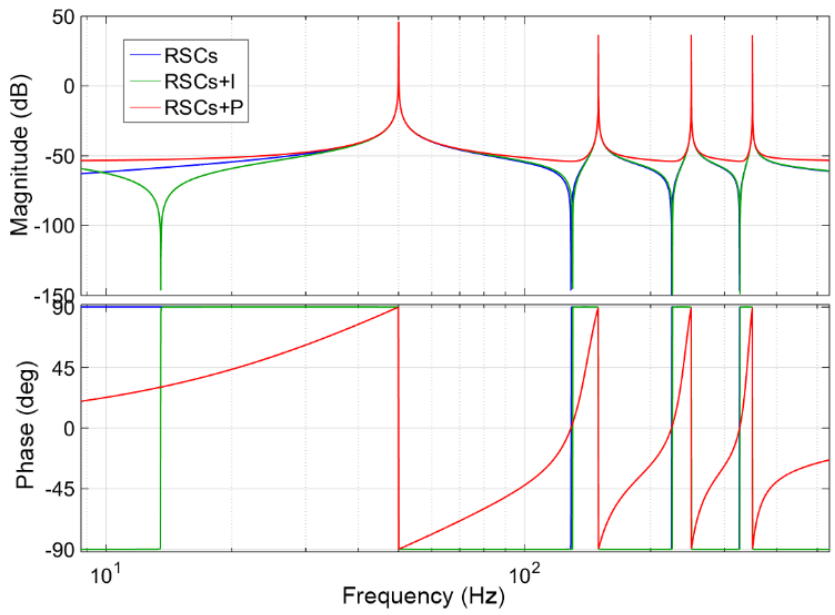

(b)

Fig. 3. a) Block scheme of different RSC based control structures. Vr: reference. Vm: measure. Dout: control signal forwarded to the modulator, b) Bode plots for different RSC based control topologies.

\section{REPETITIVE CONTROL}

Repetitive Control represents an optimal control solution for feedback systems that are subject to periodic inputs [711]. Differing from traditional approaches, a repetitive controller is not intended to stabilize the plant, but to augment an existing control system for removal of the periodic error signals. Based on the Internal Model Principle (IMP) [12], the repetitive controller processes the error signal of the previous period and applies the resultant signal to improve the control performance of the current cycle. Theoretically, with a suitably designed RC, the output of a stable feedback system can track the periodic reference signal or/and reject the exogenous periodic disturbance with zero steady state error even in the presence of model uncertainties. Based on the configuration with which they are integrated with the pre-existent stabilizing control system, repetitive controllers can be roughly divided into three main categories: direct type, parallel type and plug-in type. Fig. 4 illustrates the three configurations, where r, e, y, $\mathrm{G}_{\mathrm{P}}, \mathrm{G}_{\mathrm{C}}$ and $\mathrm{G}_{\mathrm{RC}}$ represent the periodic reference, the error signal, the system output, the plant, the stabilizing controller (which will be the resonant controller at $50 \mathrm{~Hz}$ in this work) and the repetitive controller, respectively.
In this work, the selected plug-in type RC form allows to design $\mathrm{G}_{\mathrm{C}}$ individually to stabilize the target plant $\mathrm{G}_{\mathrm{P}}$, which is particularly helpful for internally unstable and nonminimum phase systems. Fig. 5 shows the plug-in type RC feedback control system used in this paper, where the structure of the RC is highlighted in detail. $\mathrm{K}_{\mathrm{RC}}$ is the repetitive learning gain, $\mathrm{z}^{-\mathrm{M}}$ is the delay line, $\mathrm{Q}(\mathrm{z})$ is the robustness filter, and $G_{f}(z)$ is the stability filter. $M$ is the ratio between the pulse period $\mathrm{T}_{\mathrm{p}}$ and the sampling time $T_{S}$.

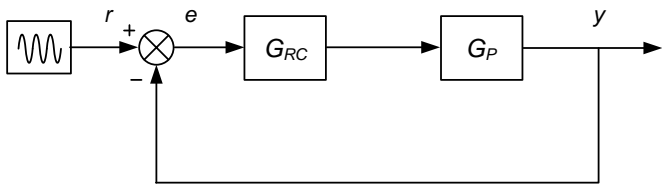

(a)

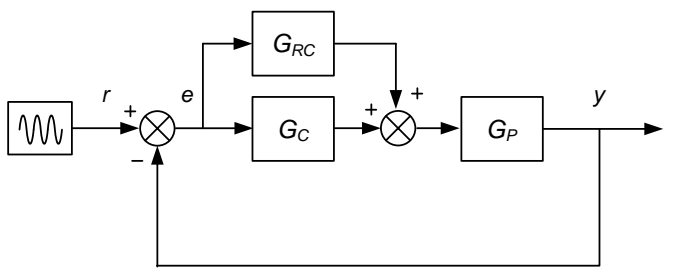

(b)

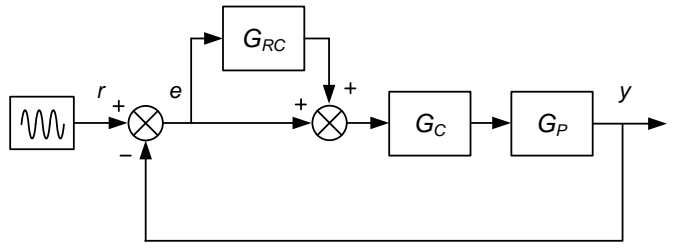

(c)

Fig. 4. Structures of Repetitive Control Systems. a) direct type, b) parallel type, c) plug-in type.

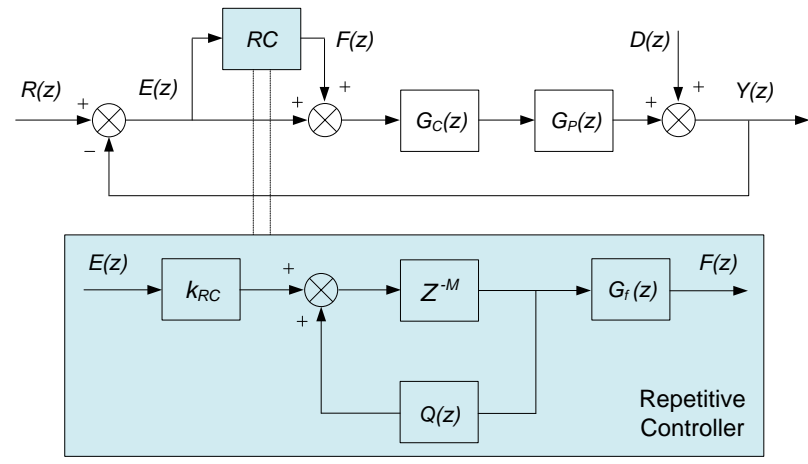

Fig 5. Plug-in Type RC control system.

The $\mathrm{RC}$ transfer function is presented in (5).

$$
\mathrm{RC}=\frac{\mathrm{F}(\mathrm{z})}{\mathrm{E}(\mathrm{z})}=\frac{\mathrm{k}_{\mathrm{RC}} \mathrm{G}_{\mathrm{f}}(\mathrm{z}) \mathrm{Z}^{-\mathrm{M}}}{1-\mathrm{Q}(\mathrm{z}) \mathrm{Z}^{-\mathrm{M}}}
$$

Taking RC into account and including the effect of periodic disturbances, the error transfer function for the overall system is expressed in (6) [8]. 


$$
\begin{aligned}
& E(z)=\frac{R(z)-D(z)}{1+(1+R C) G_{C}(z) G_{P}(z)} \\
& =\frac{(R(z)-D(z)) \cdot\left(1-Q(z) Z^{-M}\right)}{\left(1+G_{C}(z) G_{P}(z)\right) \cdot\left[1-z^{-M}\left(Q(z)-\frac{k_{R C} G_{f}(z) G_{C}(z) G_{P}(z)}{1+G_{C}(z) G_{P}(z)}\right)\right]}
\end{aligned}
$$

Based on the small gain theorem [9], two sufficient stability conditions for the plug-in RC system are derived as follows: first, the roots of $1+\mathrm{G}_{\mathrm{C}}(\mathrm{z}) \mathrm{G}_{\mathrm{P}}(\mathrm{z})$ need to remain within the unit circle; second, equation (7) is guaranteed for all frequencies below the Nyquist frequency.

$$
\left|S\left(e^{j \omega T S}\right)\right|=\left|Q\left(e^{j \omega T s}\right)-\frac{k_{R C} G_{f}\left(e^{j \omega T S}\right) G_{C}\left(e^{j \omega T s}\right) G_{P}\left(e^{j \omega T S}\right)}{1+G_{C}\left(e^{j \omega T S}\right) G_{P}\left(e^{j \omega T S}\right)}\right|<1
$$

\section{RESONANT-REPETITIVE COMBINED CONTROL}

In this work, a resonant-repetitive combined control strategy is considered for the target 3-phase 4-leg inverter system. The RSC is designed and tuned at the main frequency of $50 \mathrm{~Hz}$, which ensures not only sufficient stability margin for the system, but also a fast and smooth output dynamics before RC takes action. The aim of $\mathrm{RC}$ is to compensate higher order of harmonics cycle by cycle, and eventually achieve zero steady state error.

Complete system modeling has been accomplished according to the block scheme shown in Fig. 6 where the plant transfer function $\mathrm{G}_{\mathrm{P}}(\mathrm{s})$ is composed respectively by the model of both the 4-leg VSI, $\mathrm{G}_{4-\mathrm{leg}}(\mathrm{s})$, and the output power filter, $G_{p w f}(s) . G_{l p f}(s)$ takes into account the measurement chain as an equivalent Butterworth type II order low-pass filter having a cut-off frequency of $10 \mathrm{kHz}$. These subsystems have been modeled according to the procedure shown in [6], with reference to the inverter output filter structure depicted in Fig. 7 and having the component parameters as listed in Table I.

From [6], complete system modeling equations are briefly reported as it follows

$$
\begin{gathered}
G_{4-l e g}(s)=\frac{K_{m} V_{d c}}{1+\frac{s}{2 \pi F_{s w}}} \\
G_{l p f}(s)=\left(\frac{\omega_{f}^{2}}{s^{2}+\sqrt{2} \omega_{f} s+\omega_{f}^{2}}\right) \\
Z_{\text {trap }}(s)=s L_{t}+\frac{1}{s C_{t}}, Z_{\text {dump }}(s)=s L_{d}+\frac{1}{s C_{d}}+R_{d} \\
G_{p w f}(s)=\frac{Z_{\text {dump }}(s) / / Z_{\text {trap }}(s) / / \frac{1}{s C}}{s L_{k}+\left(Z_{\text {dump }}(s) / / Z_{\text {trap }}(s) / / \frac{1}{s C}\right)}
\end{gathered}
$$

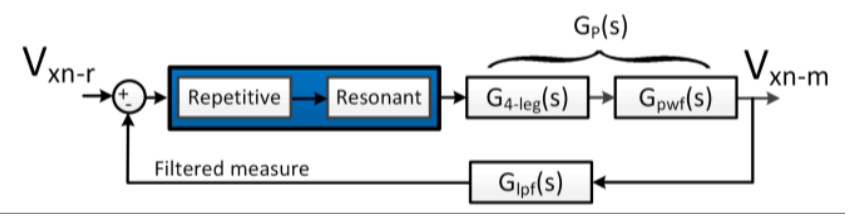

Fig. 6. Cascade configuration for the proposed RR control. x: a,b,c. r: reference voltage. m: measured output voltage. $n$ : neutral voltage.

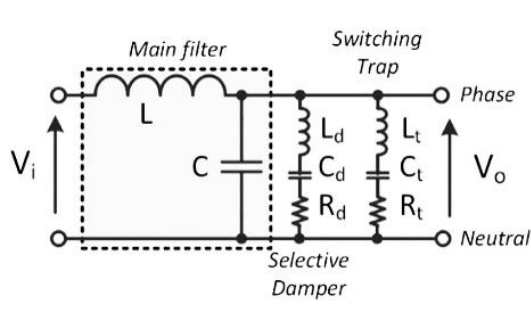

Fig. 7. Scheme of the inverter output power filter.

TABLE I OUTPUT FILTER PARAMETERS

\begin{tabular}{|c|c|}
\hline$L$ & $800 \mu \mathrm{H}$ \\
\hline$C$ & $5 \mu \mathrm{F}$ \\
\hline$L_{d}$ & $1 \mathrm{mH}$ \\
\hline$C_{d}$ & $2.2 \mu \mathrm{F}+0.47 \mu \mathrm{F}$ \\
\hline$R_{d}$ & $15 \Omega$ \\
\hline$L_{t}$ & $172 \mu \mathrm{H}$ \\
\hline$C_{t}$ & $2 \times 0.56 \mu \mathrm{F}$ \\
\hline$R_{t}$ & $50 \mathrm{~m} \Omega$ \\
\hline
\end{tabular}

where $K_{m}$ is the gain related to the modulation strategy, $V_{d c}$ and $\mathrm{F}_{\mathrm{sw}}$ are respectively the input DC-link voltage and the inverter switching frequency being assumed equal to the sampling frequency. With reference to $G_{l p f}(f), \omega_{f}$ is the filter cut-off frequency. The output power filter transfer function (11) can be straightforwardly obtained from Fig. 8 using (10) yielding directly the expression for $\mathrm{G}_{\mathrm{pwf}}(\mathrm{s})$.

The filter architecture is investigated in [13] and it is intended to be used mainly in applications for off-grid operations. The $L C_{\text {main }}$ section of the filter is a standard second order passive low-pass filter, composed by a power inductor and a high current ripple film capacitor. This basic topology is combined with one or more either LC or RLC tuned circuits, placed in parallel with the main capacitor $\mathrm{C}_{\mathrm{F}}$. In the considered filter structure, the main capacitor value has been strongly reduced with respect to conventional LC filters [14], improving the filter impedance at fundamental frequency and dramatically reducing the reactive current component.

With reference to Fig. 6, from Fig. 5 and (3), in the sdomain Resonant and Repetitive controllers can be combined as follows

$$
\begin{aligned}
G_{R R}(s) & =G_{R C}(s) G_{R S C}(s)= \\
& =\underbrace{\left(1+\frac{K_{R C} e^{-T_{1} s}}{1-Q e^{-T_{0} s}}\right)}_{\text {Repetitive }} \underbrace{\frac{2 k_{i r}\left(\omega_{c r} s+\omega_{c r}^{2}\right)}{s^{2}+2 \omega_{c r} s+\omega_{c r}^{2}+\omega_{0}^{2}}}_{\text {Resonant }}
\end{aligned}
$$

where $T_{0}$ is the main delay and $T_{1}$ is the delay related to the stability filter. The repetitive Control transfer function shown in (12) can be derived directly from the block scheme reported in Fig. 5, whereas a full Resonant Controller representation in s-domain can be written as shown in [5]. The controller parameters are tuned by means of Nyquist plot, however Bode plots or Nychols chart can be used as well. However, due to the well-known issues 
arising during the resonant controller discretization process, a separate z-domain representation is considered. According to that, the RSC has been discretized applying the bilinear transformation with frequency pre-warp, whereas the RC has been implemented by the direct delay-line expression as in (5). The achieved combined controller form in discrete domain is compact and it can be straightforwardly implemented on industrial grade DSP.

All the controller parameters are summarized in Table II.

TABLE II

PARAMETERS OF THE PROPOSED RR CONTROL SYSTEM

\begin{tabular}{|c|c|c|}
\hline Symbol & Description & Value \\
\hline $\mathrm{k}_{i r}$ & Resonant controller gain & 350 \\
\hline$\omega_{\mathrm{cr}}$ & Resonant controller width & 0.002 \\
\hline$\omega_{0}$ & Resonant controller frequency & 314 \\
\hline $\mathrm{K}_{\mathrm{RC}}$ & Repetitive controller gain & 0.5 \\
\hline $\mathrm{Q}(\mathrm{z})$ & Robustness filter & 0.98 \\
\hline $\mathrm{G}_{\mathrm{f}}(\mathrm{z})$ & Stability filter & $\mathrm{z}^{4}$ \\
\hline $\mathrm{M}$ & Delay line & 240 \\
\hline $\mathrm{f}_{\mathrm{s}}$ & Sampling frequency & $12 \mathrm{kHz}$ \\
\hline
\end{tabular}

Fig. 8 shows the Bode plot of the complete RR control system. Clearly, at the fundamental frequency and each harmonic frequency, the system has very high magnitude gain and phase shift of zero degree. This important feature implies that perfect reference tracking and/or disturbance rejection can be achieved in steady state at these frequencies. Fig. 9 depicts the locus curve of $S\left(e^{j \omega T s}\right)$ with $\mathrm{G}_{\mathrm{f}}(\mathrm{z})=\mathrm{z}^{4}, \mathrm{Q}(\mathrm{z})=0.98$, and $\mathrm{K}_{\mathrm{RC}}=0.5$ while the frequency varies. As it can be seen, the magnitude of the term $S\left(e^{\mathrm{j} \omega \mathrm{Ts}}\right)$ stays within the unity circle centered at $(0,0)$ for the entire frequency range up to the Nyquist Frequency $\omega_{\text {nyq }}$, so that the proposed RR system is proved to be stable.

The resulting Bode plot, for the complete system including RR control, VSI and output filter, is shown in Fig. 10 , where the high gain provided by the combined control structure can be identified at the fundamental frequency. Lower and decreasing gains are related to the Repetitive Controller filtered by the right side band of the Resonant Controller, which therefore also substitutes the effect of the robustness filter $\mathrm{Q}(\mathrm{z})$ chosen as a constant in our design. It can be noticed that around $1.7 \mathrm{kHz}$ harmonics gain moderately increases due to the resonance of the output power filter.

In the proposed plug-in structure, the RSC component is used to assure prompt tracking of the inverter output voltage as well to achieve as fast as possible system response to load variations; to this purpose, it is tuned at the fundamental frequency. RSC acts as a low-pass filter when combined with the RC. It means that high frequency gains that are typical of the RC are reduced by the RSC without the need of an additional stabilizing output low-pass filter.

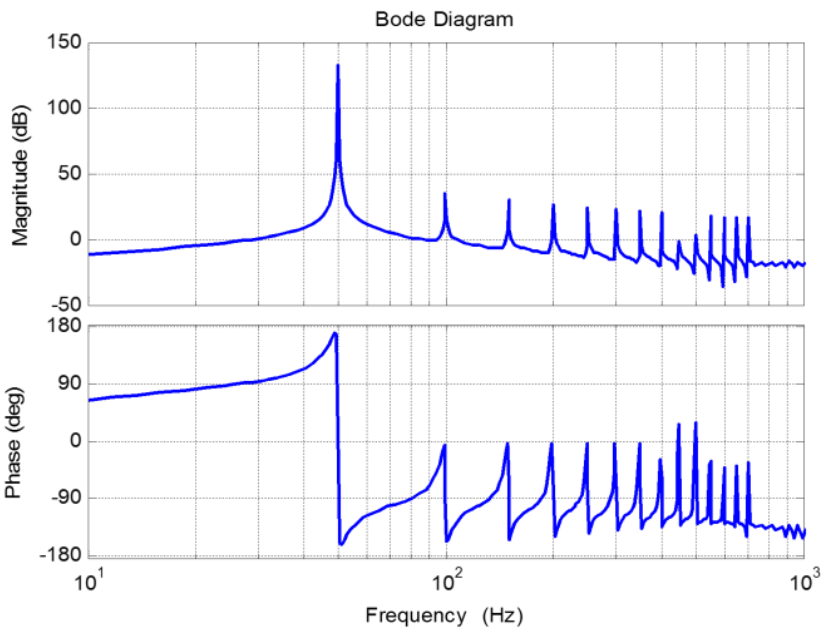

Fig. 8. Bode plot of the complete RR control system.

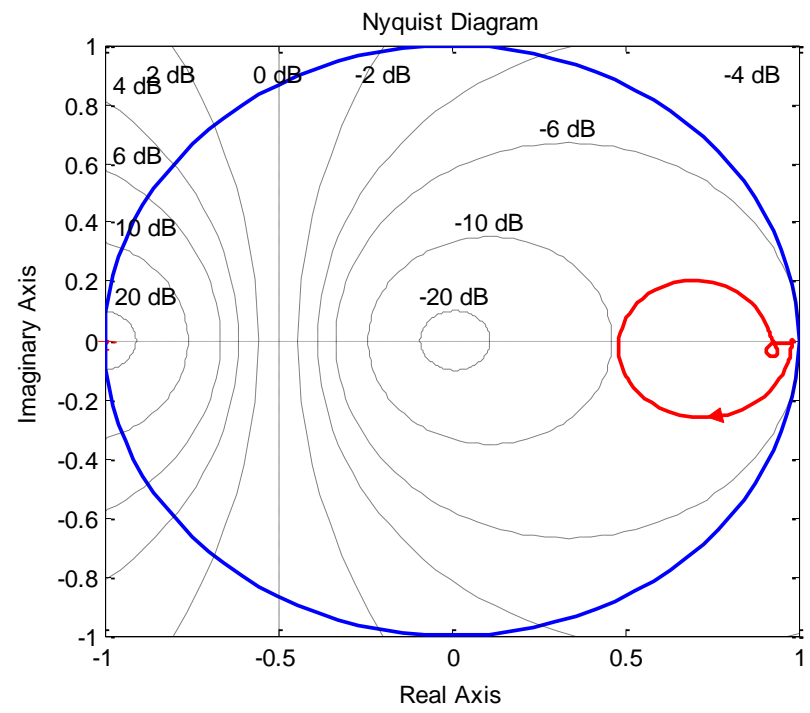

Fig. 9. Nyquist locus curve of $S\left(e^{\mathrm{j} \omega \mathrm{Ts}}\right)$ with $\mathrm{G}_{\mathrm{f}}(\mathrm{z})=\mathrm{z}^{4}, \mathrm{Q}(\mathrm{z})=0.98$ and $\mathrm{K}_{\mathrm{RC}}=0.5$.

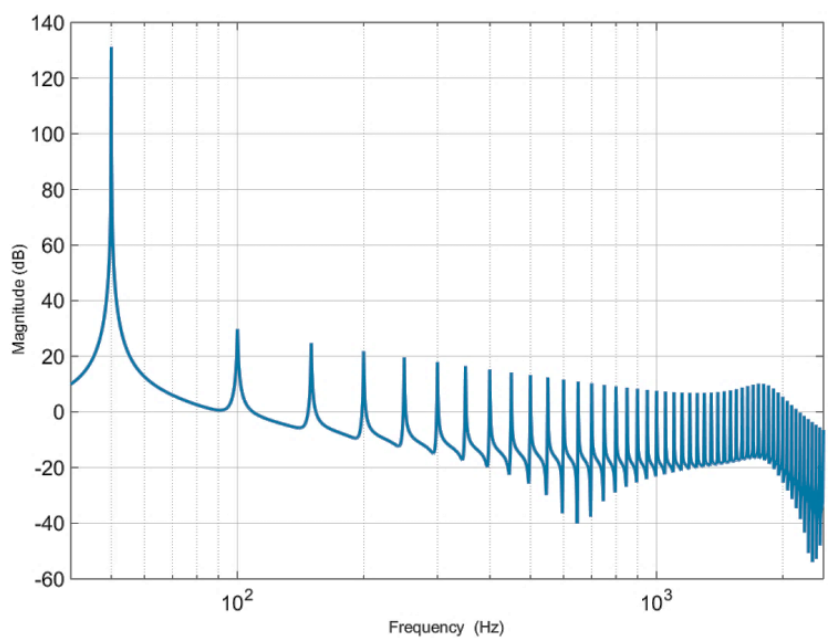

Fig. 10. Open-loop Bode plot for the complete system including RR control, VSI and output power filter. 
Repetitive-Resonant control and its main competitor Multi-Resonant structure show similar control performances being both based on the Internal Model Principle, however, some differences can be highlighted. As opposed to a fully multi-resonant control solution, the proposed resonantrepetitive combined control strategy presents less stability issues, it is more robust to system parameters drift, it can compensate in a more efficient way the high order harmonics, as well its implementation on digital control platform is much easier than multi-RSC structure.

RR performances in harmonic compensation are limited by the required high-frequency attenuation to be provided to guarantee system stability. Multi-RSC shows a different implementation being based on a parallel tank of single resonant controllers. In industrial grade control platforms, the implementation of such a number of RSCs might oblige to increase the control algorithm sampling time due to the sequential execution of a large number of mathematical operations. Increasing the the sampling time, forces also to limit the number of RSCs that can be included in the MultiRSC structure, resulting in reduced harmonic compensation capability. In conclusion, RR strategy allows a simpler implementation with strongly reduced computational burden and lowering the processor requirements, resulting in a reduced cost of the dedicated control platform, as it is described in the last Section of the paper.

\section{A. Considerations on Resonant-Repetitive and Repetitive- Integral controls}

Repetitive Control structures can be indifferently stabilized by an integral controller or, as previously mentioned, by a RSC at the fundamental frequency. However, Fig. 11a and Fig. 11b depict the different behavior at low frequency for the resulting controllers with same high frequency performance. The RR control shows higher gain in the neighborhood of the fundamental frequency with respect to the RC plus integral structure. When the comparison is made considering the same high frequency attenuation, the low gain exhibited by the Resonant-Integral (RI) controller at the fundamental frequency limits the tracking behavior with respect to reference variations, which can occur as an example during the VSI start-up procedure. Accordingly, in order to increase the gain at low frequencies, the integral action must be enhanced by increasing its gain. It results, of course, also in the amplifications of the high frequency resonances characteristics of the $\mathrm{RC}$, thus reducing the stability margins.

When Repetitive Controller is combined with the resonant control structure, the resulting configuration allows more tuning flexibility. It provides the capability to regulate independently both the total gain at the fundamental frequency, and the required attenuation at high frequencies, by changing the RSC gain $\left(\mathrm{k}_{\mathrm{ir}}\right)$ and width $\left(\omega_{\mathrm{cr}}\right)$.

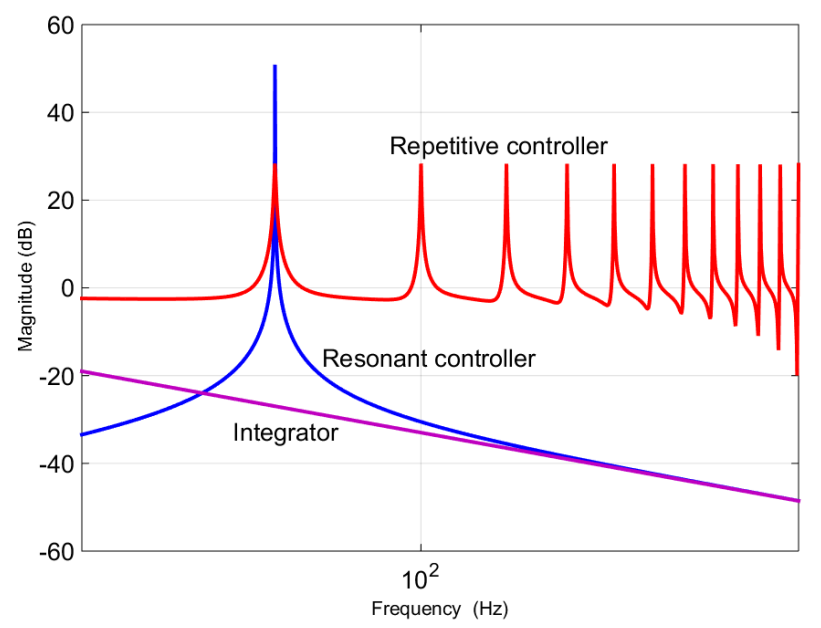

(a)

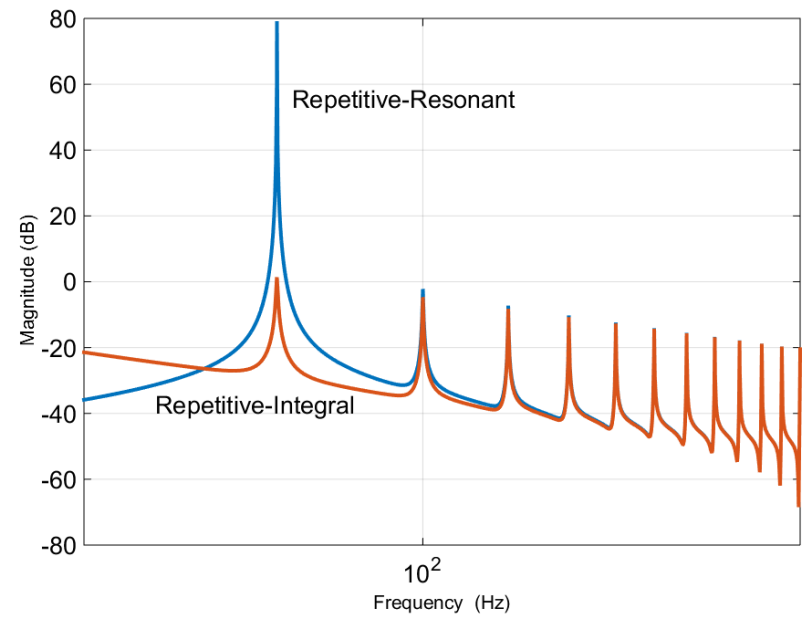

(b)

Fig. 11. Bode plot of the Resonant, Repetitive and Integral controllers. a) Repetitive, Resonant and Integral controllers, b) Repetitive-Resonant and Repetitive-Integral.

In addition, the proposed RR structure does not significantly increase the computational weight being required for the implementation, as it results from the investigation in Section VIII.

RR control is now compared to the RI solution; for this reason, two values of the integral gain are selected. The first one (RI1) is conceived in order to provide the same filtering action of the RSC, as it is shown in Fig. 11a; the second tuned RI (RI2) exhibits a higher integral gain than RI1 at the fundamental frequency. This second solution has been motivated by the need to improve the poor tracking performance of RI1 at fundamental frequency. Fig. 12a depicts the previously described behavior showing magnitude and phase Bode plots in the neighborhood of the fundamental frequency. It can be noticed how the RR provides better tracking capability and selectivity with respect to the RI1 and RI2 solutions. Further information can be obtained analysing the Bode plots at higher frequencies, as it is shown in Fig. 12b. The use of RSC or 
Integral action does not affect the resulting phase behavior, as it is depicted in Fig. 12b; however, important differences are present in the magnitude of the analyzed controller solutions. The RI2 configuration is clearly not stable at frequencies even lower than $1 \mathrm{kHz}$. The RI1 controller is almost at the boundary of the stability condition with a very small gain margin. The proposed RR controller provides higher stability margins than the previously mentioned solutions. Jointly with the better tracking capabilities at the fundamental frequency, it offers also harmonic compensations and control chain stabilization.

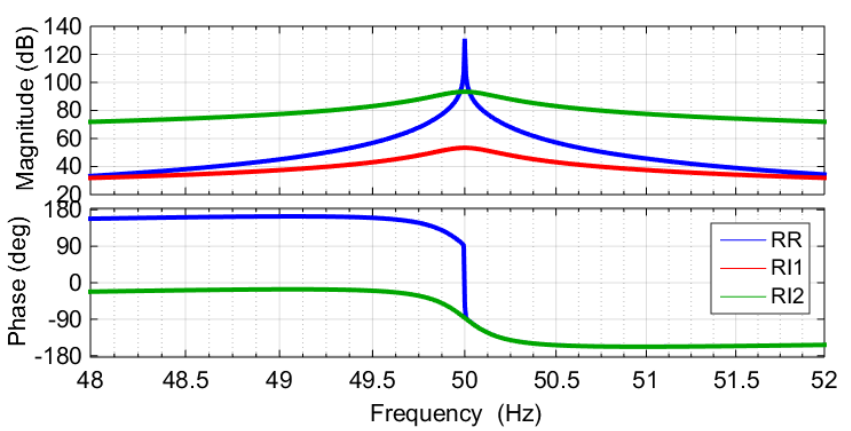

(a)

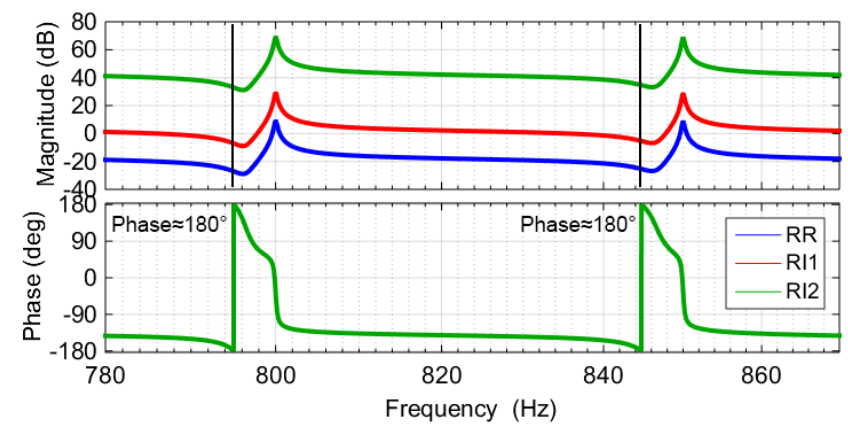

(b)

Fig. 12. Open-loop Bode plots of the complete system. a) centered across the fundamental harmonic, b) zoom across higher order harmonics.

\section{Simulation Results}

First simulation results for the RR control structure have been obtained through the linearized model, in which the switching behavior is neglected. In this case Fig. 13 depicts both RC and combined RR control signals when no harmonic content affects the $230 \mathrm{~V}_{\text {rms }}$ phase-to-neutral output voltage; Fig. 14 shows the same control signals in case of output voltage harmonic content of $11 \%, 10 \%$ and $9 \%$ respectively for $3^{\text {rd }}, 5^{\text {th }}$ and $7^{\text {th }}$ harmonic order.

The proposed RR configuration is capable of sharing the control effort between RC and RSC. In fact, RSC is devoted to provide tracking of the fundamental harmonic, whereas $\mathrm{RC}$ is used to cancel the output voltages harmonic contents introduced by distortive loads.

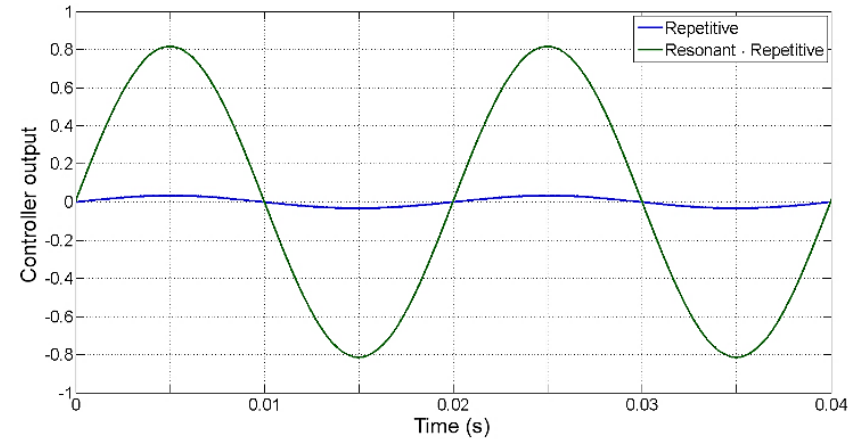

(a)

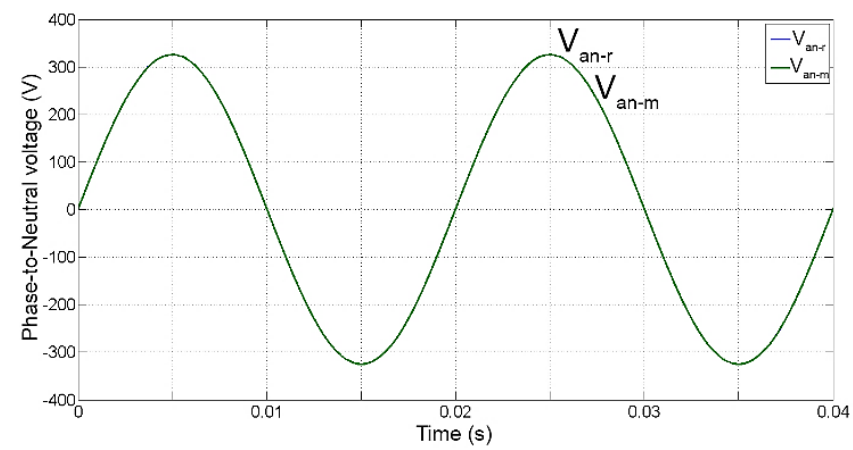

(b)

Fig. 13. Simulation Results with no harmonic content. a) RC and RR Signals, b) Phase-to-Neutral Output Voltage.

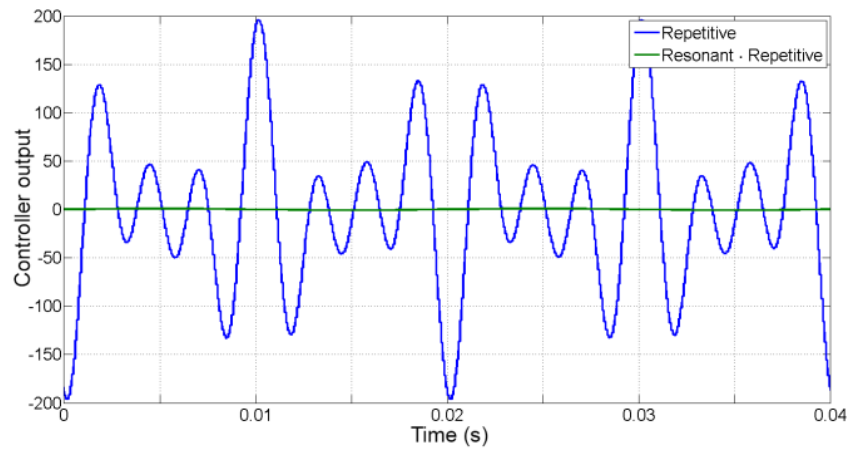

(a)

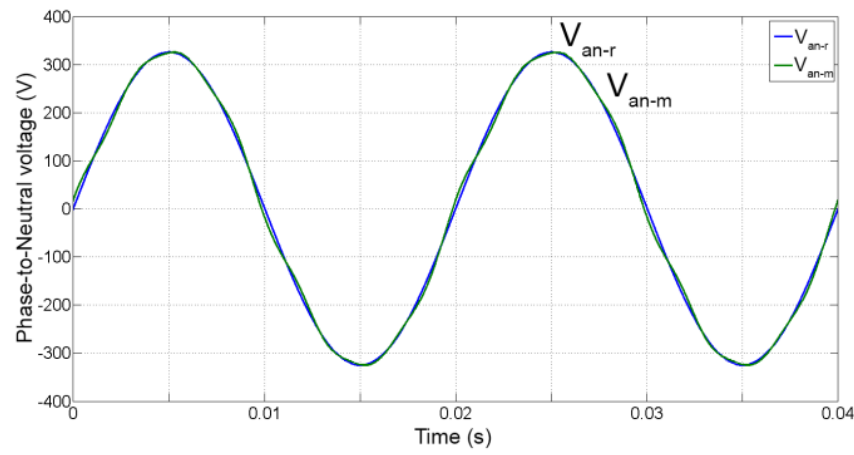

(b)

Fig. 14. Simulation Results with harmonic content. a) RC and RR Signals, b) Phase-to-Neutral Output Voltage. 
Comprehensive analysis has been performed through a full switching model, which includes the single-precision floating-point 32-bit arithmetic, as in the selected Digital Signal Processor (DSP), synchronized sampling and PWM unit. The IGBT Dead-Time is equal to $3.3 \mu \mathrm{s}$, and the modulation scheme is selected to be the Carrier based PWM with triangular third harmonic injection. The proposed RR control is designed to have high performance in output voltage regulation for both highly non-linear loads and heavy load variations. In order to highlight the behavior of the RR control, in the case of highly non-linear loads, test results are obtained when a three-phase diode rectifier load is fed by the 4leg VSI, as it is depicted in Fig. 15 and Fig. 16.

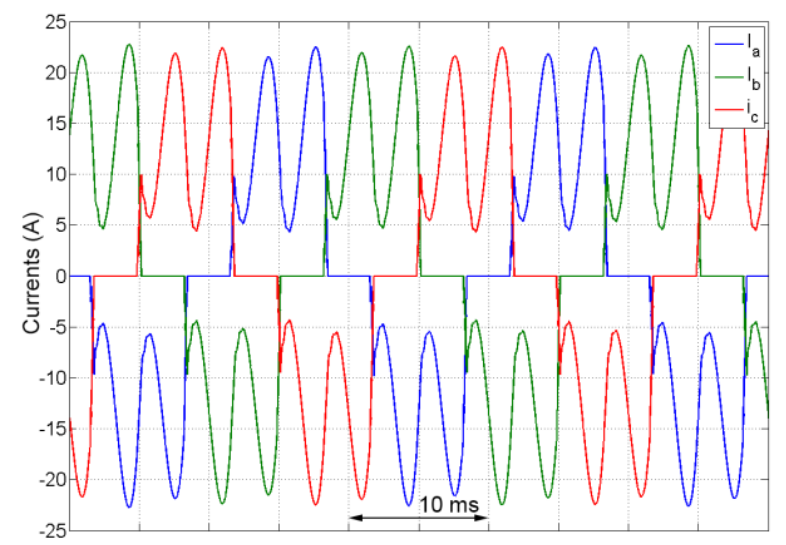

Fig. 15. Inverter output currents for the 3 ph diode rectifier load.

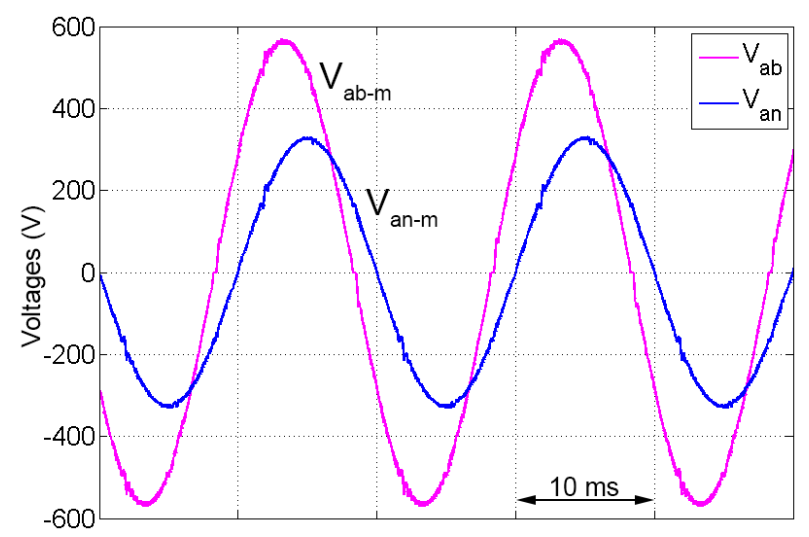

Fig. 16. Inverter output line-to-line voltage and phase voltage for the $3 \mathrm{ph}$ diode rectifier load with RR control structure fully enabled.

The non-linear load consists of a three-phase diode bridge rectifier configuration having $0.8 \mathrm{mH}$ as input line inductance, $1.5 \mathrm{mF}$ as output DC capacitance and $25 \Omega$ as DC load. Fig. 15 depicts the heavily distorted load currents. However, thanks to the effect of the RR algorithm, inverter output line-to-line voltage and phase voltage exhibit a quite low harmonic distortion as shown in Fig. 16, with a resulting THD around $1.7 \%$. A similar simulation test is performed when the inverter is loaded by a single-phase diode rectifier, as it is shown in Fig. 17 and Fig. 18 respectively for the output currents and phase voltages. The sinusoidal output voltage shaping performed by the RR control structure provides a very low THD even in case of highly distorted and unbalanced currents.

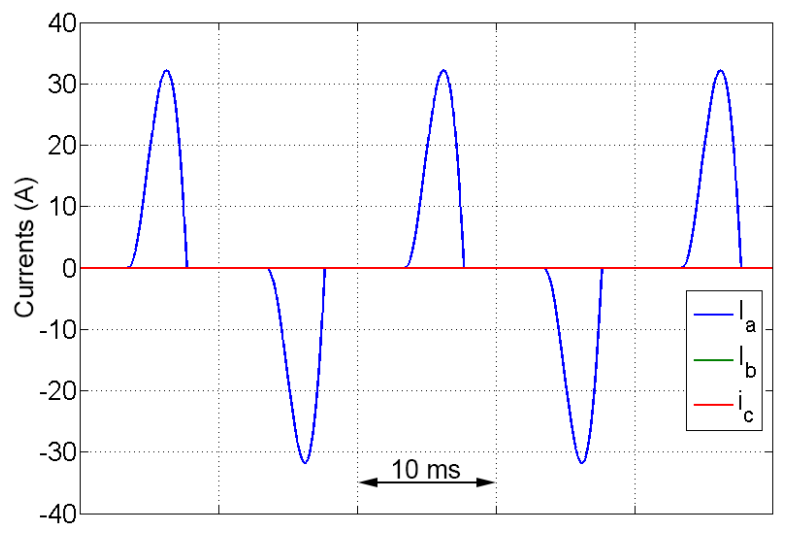

Fig. 17. Inverter output currents for the $1 \mathrm{ph}$ diode rectifier load

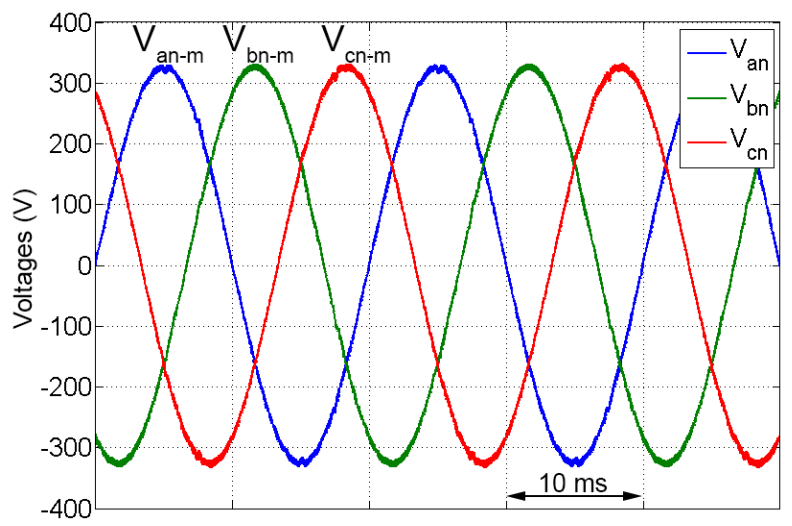

Fig. 18. Inverter output phase voltages for the $1 \mathrm{ph}$ diode rectifier load with RR control structure fully enabled.

\section{EXPERIMENTAL TESTS}

Experimental tests have been performed to validate the proposed Resonant-Repetitive combined control architecture with parameters' values as in Table II.

Prototypal realization of a 3-phase 4-leg inverter as shown in Fig. 19 is used to experimentally investigate the resonant-repetitive combined control. The prototype is rated $40 \mathrm{kVA}$ at $50{ }^{\circ} \mathrm{C}$ ambient temperature and its characteristics are listed in Table III. It is able to supply power to electric utility systems with extremely unbalanced loads up to 13.3 $\mathrm{kVA}$ rated power per phase, as well highly distorted loads as single-phase diode rectifier and very low power factor utilities as induction motors at starting operation.

As already mentioned in the simulations Section, the selected modulation scheme is the Carrier based PWM with triangular third harmonic injection.

The test bench as in Fig. 20 has been set up in order to carry out the experimental tests. Three different load conditions have been considered: three-phase non-linear 
load for harmonic content evaluation, single-phase linear load for unbalanced load as well for load transient conditions, three-phase loads with variable power factor.

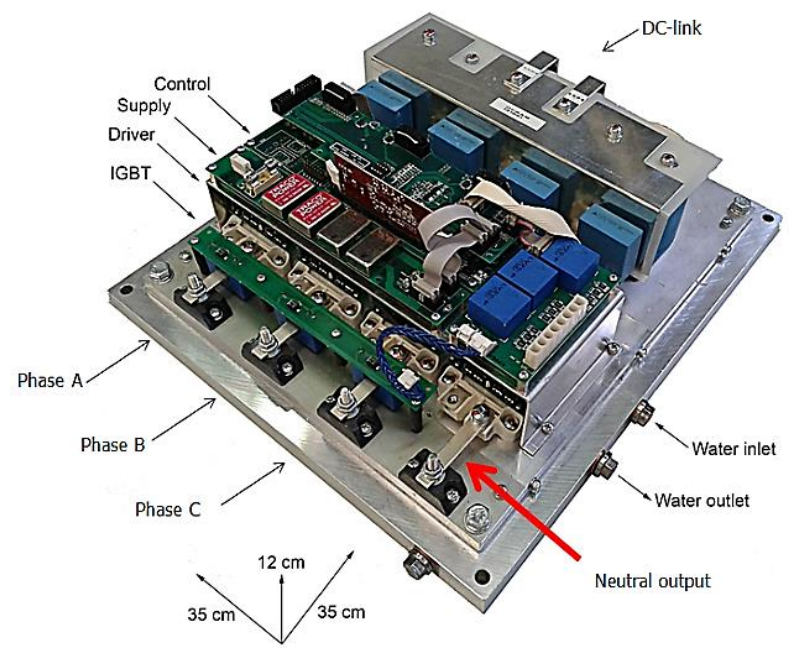

Fig. 19. 3-Phase 4-Leg Inverter prototype.

TABLE III

3-PHASe 4-Leg InVERTER PROTOTYPe MAIN CHARACTERISTICS

\begin{tabular}{|l|l|}
\hline Rated Power & $40 \mathrm{kVA}$ \\
\hline Line-to-Line Voltage & $400 \pm 10 \% \mathrm{~V}$ \\
\hline Switching Frequency & $12 \mathrm{kHz}$ \\
\hline $\begin{array}{l}\text { Efficiency @ rated power } \\
\text { (output filter included) }\end{array}$ & 0.97 \\
\hline Power Modules & Semikron - SEMIX303GB12Vs \\
\hline Snubber Capacitors & $\mathrm{MKP} 1.2 \mu \mathrm{F}-1000 \mathrm{Vdc}$ \\
\hline DC-Link Capacitors & $\mathrm{MKP} 450 \mu \mathrm{F}-900 \mathrm{Vdc}$ \\
\hline AC Output Inductors & $800 \mu \mathrm{H}$ \\
\hline AC Output Main Capacitors & $\mathrm{MKP} 5 \mu \mathrm{F}-480 \mathrm{Vac}$ \\
\hline Control Board & $\mathrm{TMS} 320 \mathrm{~F} 28335$ \\
\hline
\end{tabular}

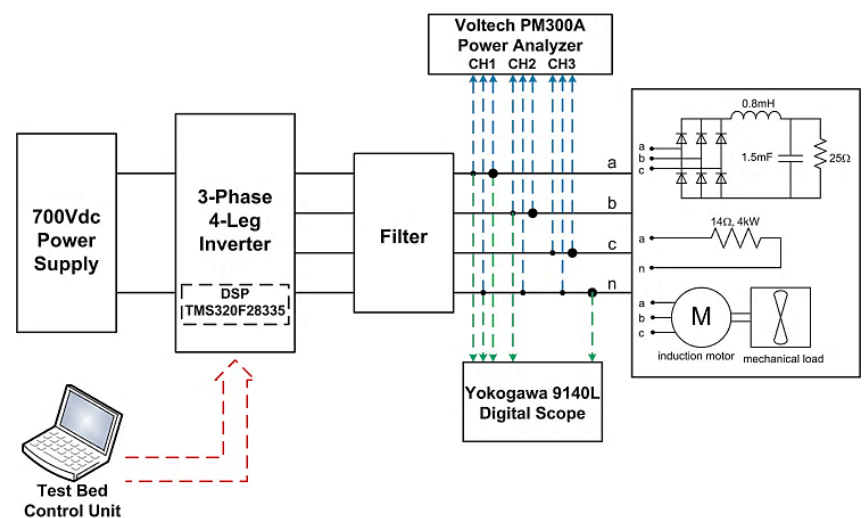

Fig. 20. Test Bench layout

A non-linear load has been arranged to test the system under extreme power quality conditions. As in the simulation tests, the non-linear load consists of a threephase diode bridge rectifier configuration having $0.8 \mathrm{mH}$ as input line inductance, $1.5 \mathrm{mF}$ as output DC capacitance and $25 \Omega$ as resistive DC load.
At steady state mode of operation, inverter line-to-line voltage, phase voltage and output current are illustrated in Fig. 21 when the described non-linear load is applied. Output voltage THD is about $1.7 \%$ when the current THD amounts to $48 \%$.

With the proposed control solution, algorithms for compensation of switching devices Dead-Time (DT) are not required. In fact, in the experimental results shown in this work, the usual distortive effects introduced by DT are negligible even when the inverter DT compensation is disabled. DT introduces mainly fifth and seventh harmonics distortion, which is perfectly compensated by the repetitive control section, thus achieving a simplification in the whole control algorithm.

Dynamic performances of the investigated RR control have been preliminary tested through a load step test in extreme unbalanced conditions. A single-phase resistor having rated power of $4 \mathrm{~kW}$ at $230 \mathrm{Vrms}$ is used as load step test. The resistive load is connected to the output of the inverter when the phase-to-neutral inverter voltage reaches its peak value. As it is shown in Fig. 22, both line-to-line and phase-to-neutral voltage drops are quite negligible due to the fast compensation provided by the implemented RR control.

As soon as the load is switched on, output voltages exhibit some distortion, due to the time required by the $\mathrm{RC}$ to compensate VSI filter voltage drops and DT effects. The amount of time required to reach the steady state THD value of $1 \%$ in the output voltage is around eight fundamental periods, which is equal to $160 \mathrm{~ms}$ considering EU frequency standard.

Experimental testing has been also carried out in case of reactive power loads and the relevant results are shown in Fig. 23. A three-phase induction motor is supplied at partial load operating conditions, in order to achieve a 0.6 power factor load condition.

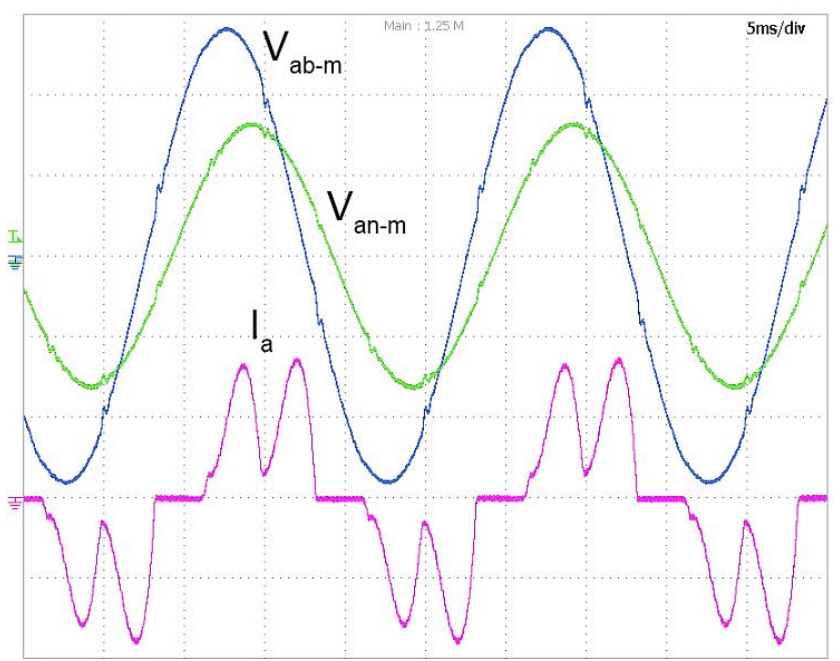

Fig. 21. Experimental results for a 3ph diode rectifier load. Voltage 200 V/div, current $20 \mathrm{~A} /$ div. 


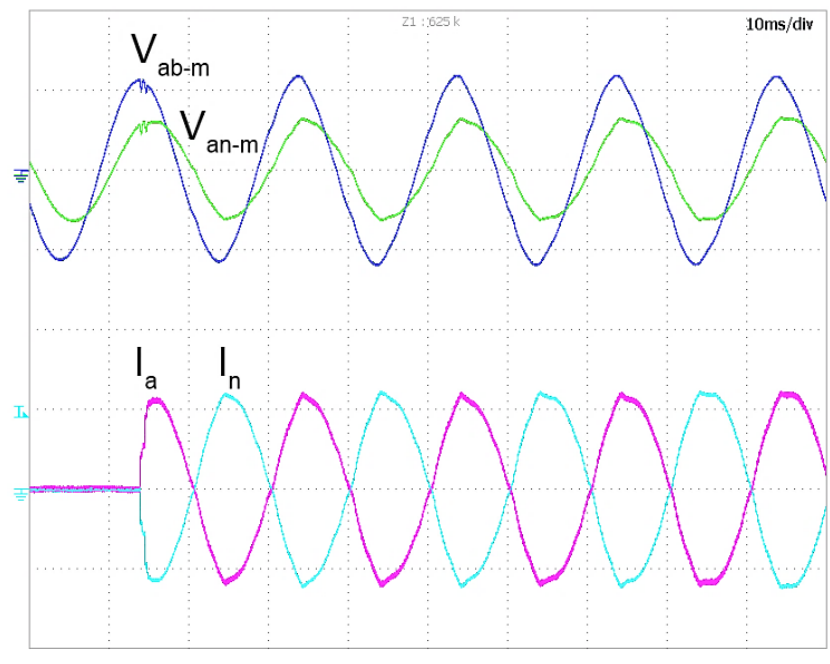

Fig. 22. Experimental results for a single phase resistive load step from noload to $4 \mathrm{~kW}$. Voltage $500 \mathrm{~V} / \mathrm{div}$, current $20 \mathrm{~A} / \mathrm{div}$.

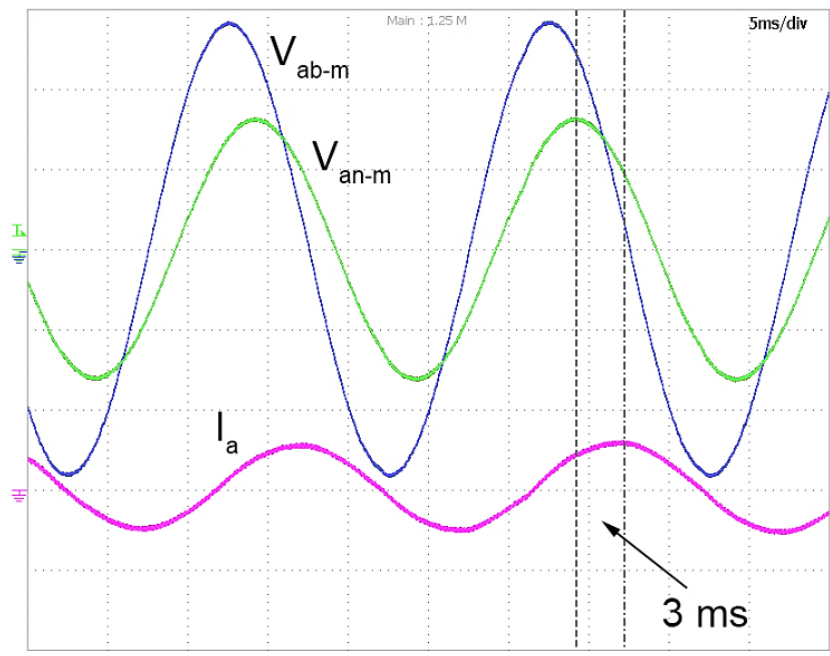

Fig. 23. Experimental results for three phase load with 0.6 power factor. Voltage $200 \mathrm{~V} / \mathrm{div}$, current $20 \mathrm{~A} /$ div.

\section{DisCUSSION ON DATA AND PROGRAM MEMORY REQUIREMENTS}

Memory requirements for the proposed RR control versus both the multi resonant control configuration and conventional RC stabilized by a simple integrator are analyzed, compared and contrasted. A common implementation benchmark is to consider 32-bit singleprecision floating-point arithmetic for all the topologies and per phase necessity. These analysis results are summarized in Table IV.

A single RSC discretized by trapezoidal approximation with frequency pre-warp requires 11 variables to be stored in data memory as well 5 multiplications, 4 additions/subtractions and 4 assignments.

$\mathrm{RC}$ needs two delay lines with length dependent on the ratio between the sampling and the fundamental frequencies. According to the value of $12 \mathrm{kHz}$ for the sampling frequency and the fundamental frequency of 50 $\mathrm{Hz}, \mathrm{RC}$ data memory requests are 240 and 236 delay lines with 2 single length temporary variables and a pair of 16 bits variables for indexing. Moreover, 2 multiplications, 2 sum operations, 2 increments, 2 comparisons and 6 assignments need to be stored in the program memory.

Considering the Multi-RSC up to the $50^{\text {th }}$ harmonic, that is usually required to be compliant with EMC 61000 Class1 standard, data memory occupancy is around 2200 bytes per phase. On the contrary, the investigated RR needs around 1970 bytes per phase; this means almost $11 \%$ reduction in data memory occupancy. When program memory requirements are considered, RR needs a total 27 operations to be executed, with different weights in memory. However, the considered Multi-RSC has to store 650 operating codes in the program memory. According to that, the proposed combined RR algorithm execution time is substantially lower than a Multi-RSC, being executed through fewer operations with respect to the RSC, in which the operations must be sequentially executed.

Data and program memory requirements of a classical Repetitive Control stabilized by a simple Integrator (RI) have been counted. The proposed RR structure do not significantly affect the memory and computational burden requirements of the conventional $\mathrm{RC}$ configuration, as reported in Table IV. The small difference in memory requirements is mainly related to the z-domain implementation of the Integrator in RI with respect to the Resonant Controller of the RR strategy, which requires a little bit more instructions being a second order system.

TABLE IV

AlGORITHM MEMORY REQUIREMENTS (PER PHASE)

\begin{tabular}{|c|c|c|}
\hline & $\begin{array}{c}\text { Data memory } \\
{[\text { bytes }]}\end{array}$ & $\begin{array}{c}\text { Program memory } \\
{[\text { instructions] }}\end{array}$ \\
\hline RR & $\approx 1970$ & $\approx 27$ \\
\hline Multi-RSC $\left(50^{\text {th }}\right)$ & $\approx 2200$ & $\approx 650$ \\
\hline RI & $\approx 1967$ & $\approx 25$ \\
\hline
\end{tabular}

\section{CONCLUSIONS}

A combined Repetitive-Resonant (RR) control algorithm for power electronics systems is investigated in this research work, using as validation benchmark a $40 \mathrm{kVA}$ stand-alone 4-leg AC power supply unit. The proposed control structure is validated with both simulation and experimental tests. Results show the excellent performance obtained by this control solution, in terms of output power quality, control dynamics, computational requirements and robustness. The use of a resonant filter tuned at the system fundamental frequency provides both system stabilization and excellent fundamental component tracking; a plug-in repetitive controller compensates for the harmonics in the controlled variable and allows the gain of the resonant filter to be 
increased for better compensation, without affecting system stability and robustness. The system performance has been tested in case of three different load conditions: 3-phase non-linear load for harmonic content evaluation, 1-phase linear load for unbalanced load as well for load transient conditions, 3-phase loads with variable power factor.

\section{REFERENCES}

[1] S. Bifaretti, A. Lidozzi, L. Solero, F. Crescimbini, "Comparison of Modulation Techniques for Active Split DC-Bus Three-Phase FourLeg Inverters", Energy Conversion Congress and Expositions, IEEE Energy Conversion Congress and Exposition ECCE 2014, Sept. 2014, pp. 5631-5638.

[2] Yunhu Yang, Keliang Zhou, Ming Cheng, "Phase Compensation Resonant Controller for PWM Converters", Industrial Informatics, IEEE Transactions on, vol. 9, no. 2, pp. 957-964, May 2013.

[3] B. Zhang, D. Wang, K. Zhou, and Y. Wang, "Linear Phase Lead Compensation Repetitive Control of a CVCF PWM Inverter", Industrial Electronics, IEEE Transactions on, vol. 55, pp. 1595-1602, 2008.

[4] L.F. Alves Pereira, J. Vieira Flores, G. Bonan, D. Ferreira Coutinho, J.M. Gomes da Silva Junior, "Multiple Resonant Controllers for Uninterruptible Power Supplies-A Systematic Robust Control Design Approach", Industrial Electronics, IEEE Transactions on, vol.61, no.3, pp.1528,1538, March 2014.

[5] Lidozzi, A.; Solero, L.; Bifaretti, S.; Crescimbini, F., "Sinusoidal Voltage Shaping of Inverter Equipped Stand-Alone Generating Units," Industrial Electronics, IEEE Transactions on, IEEE Early Access Articles. doi: 10.1109/TIE.2014.2370939
[6] A. Lidozzi, G. Lo Calzo, L. Solero, F. Crescimbini, "Integral-resonant control for stand-alone voltage source inverters," Power Electronics, IET, vol.7, no.2, pp.271,278, February 2014

[7] K. Zhou, D. Wang, "Digital repetitive controlled three-phase PWM rectifier", Power Electronics, IEEE Transactions on, vol. 18, pp. 309316, 2003.

[8] S. Chen, Y. M. Lai, S. C. Tan, and C. K. Tse, "Analysis and design of repetitive controller for harmonic elimination in PWM voltage source inverter systems", Power Electronics, IET, vol. 1, pp. 497-506, 2008.

[9] P. Mattavelli, L. Tubiana, and M. Zigliotto, "Torque-ripple reduction in PM synchronous motor drives using repetitive current control", Power Electronics, IEEE Transactions on, vol. 20, pp. 1423-1431, 2005.

[10] Y. He, J. Liu, Z. Wang, and Y. Zou, "An Improved Repetitive Control for Active Power Filters with Three-Level NPC Inverter", in Applied Power Electronics Conference and Exposition, 2009. APEC 2009. Twenty-Fourth Annual IEEE, 2009, pp. 1583-1588.

[11] Chao Ji; Zanchetta, P.; Carastro, F.; Clare, J., "Repetitive Control for High-Performance Resonant Pulsed Power Supply in Radio Frequency Applications," Industry Applications, IEEE Transactions on , vol.50, no.4, pp.2660,2670, July-Aug. 2014.

[12] B. A. Francis, and W. M. Wonham, "The internal model principle of control theory", Automatica, vol. 12, pp. 457-465, 1976.

[13] G. Lo Calzo, A. Lidozzi, L. Solero, F. Crescimbini, "LC filter design for on-grid and off-grid distributed generating units", IEEE Trans. Ind. Applicat., IEEE Early Access Articles DOI 10.1109/TIA.2014.2345952.

[14] Jinwei He, Yun Wei Li, "Generalized Closed-Loop Control Schemes with Embedded Virtual Impedances for Voltage Source Converters with LC or LCL Filters", Power Electronics, IEEE Transactions on, vol. 27, no.4, pp.1850-1861, April 2012. 